DOI: $10.3901 / J M E .2013 .07 .113$

\title{
摇臂转向架式星球车单索重力补偿*
}

\author{
刘 振 高海波 邓宗全 陶建国 \\ (哈尔滨工业大学机电工程学院 哈尔滨 150080)
}

\begin{abstract}
摘要: 为简单、准确地进行摇臂转向架式星球车(简称星球车)低重力模拟, 研究其单索重力补偿的问题。提出使用单根补偿 吊索和摇臂配重模拟星球车所有姿态下全部车轮地面接触力的方法。以四、六轮星球车为例, 建立崎岖地型上轮地接触力模 型; 通过分析模型的解结构, 证明 “正确补偿接触力” 等价于 “正确补偿狭义接触力(全部接触力水平分量为零时的特例)”; 给出吊索和配重(补偿力系)的施加方法; 通过证明补偿后的狭义接触力与低重力环境中一致, 证明补偿方法正确。仿真结果 验证了此方法的优势和正确性。针对向车厢(封闭体)内部点施加补偿力的问题, 提出相似悬挂原理: 利用悬挂体和封闭体上 关节点、受力点的全等位置关系, 使连接于悬挂体上的吊索始终向封闭体内部点施加等效补偿力。为避免拆解星球车进行质 心测量, 提出使用不同姿态下的狭义接触力计算、校正补偿力系的方法; 相应地, 给出狭义接触力的测量原理和测量平台结 构。实践证明, 研究为星球车的低重力模拟提供了简单、精确的方案原理和方法。
\end{abstract}

关键词: 摇臂转向架式星球车 低重力 重力补偿 轮压

中图分类号: V21 TH11

\section{Gravity Compensation for Rocker-bogie Rovers Through Single String Tension}

\author{
LIU Zhen GAO Haibo DENG Zongquan TAO Jianguo \\ (School of Mechanical Engineering, Harbin Institute of Technology, Harbin 150080)
}

\begin{abstract}
The crucial problems encountered in gravity compensation (GC) for rocker-bogie rovers through single string tension are examined and tackled to enable easy and precise low gravity simulation. A GC approach utilizing single string and counterweights on rockers is developed to simulate all the wheel contact forces of rovers in all configurations. The contact force model of four and six wheeled rovers is constructed, through which the equivalence between ensuring contact force and defined contact force (wheel pressure), the special case when the horizontal components of all contact force is nil, is proved. The approach is proved by showing compensated wheel pressure is identical with that in low gravity. The advantage and validity of such approach is future proved by software simulation. To equivalently apply compensation force onto the inner point of the carriage (a sealed body), the similarly suspension mechanism is developed. The mechanism is such that the relative position between surface points and inner points of the sealed body is exactly identical with those of the suspension body; compensation force on the suspension body is equivalently applied to corresponding inner point of the sealed body. To avoid dismantling of rover for the center of mass measurement, an algorithm is established to relate the position and magnitude of compensation force system with wheel pressure. The method and equipment are established to measure the wheel pressure. These researches provide a precise and feasible plan for rocker-bogie rovers' gravity compensation, which is proved by their application in experiment.
\end{abstract}

Key words: Rocker-bogie planetary rover Low gravity Gravity compensation Wheel pressure

0 前言

为消除星球车地面测试中车轮沉陷量过大、滑

* 国家自然科学基金(50975059, 61005080)、科技部-国际科技合作 (2010DFR70270)和 “111” 工程(B07018)资助项目。20120712 收到初 稿, 20121220 收到修改稿
转率过高和动力配置不足等假象，需要进行低重力 模拟试验。试验包含两部分: (1) 模拟车轮与地面接 触力; (2) 模拟月壤参数, 后者不在本文讨论范围。 星球车低重力模拟方法有三类。第一类是抛物 线飞行法。使用此方法日本学者发现了单圆柱轮沙 槽试验中重力场对沙土的影响 ${ }^{[1]}$ 。Micro5 星球车缩 比模型的低重力模拟试验 ${ }^{[2]}$ 和 LSSM 型载人月球车 
的低重力运动性能试验 ${ }^{[3]}$ 也在抛物线飞行过程中完 成。这种方法模拟效果真实，但对试验空间、时间 的限制也很明显。

第二类方法是构造低质量样车进行试验。此方 法在履带式、无悬架式和弹簧悬架式星球车上有较 多应用 ${ }^{[4]}$ 。由于摇臂式星球车减重裕量有限, 精神 号火星探测车的低质量样车只能略小于正样质量的 一半 ${ }^{[5]}$ 。另外, 摇臂构件(摇臂和与其相连的车轮组 成的构件)减重裕量很小, 所以构件质心相对旋转点 的转矩无法被降低, 因而造成轮地接触力分配失真。 模拟重力场越低, 模拟误差越大。这一点在仿真章 节中进行验证。

第三类方法是使用吊索向星球车施加补偿力 (重力补偿), 分为多索和单索两类。多索补偿方法 的补偿效果准确, 但是工程实施复杂。洛克希德公 司为美国国家航空航天局载人月球车设计了多吊索 重力补偿系统方案 ${ }^{[6]}$ 。系统的每根吊索通过单独的 拉力伺服系统维持恒拉力; 为保持吊索垂直, 位置 伺服系统跟踪月球车在水平面内的平移和转动; 由 于不能跟踪车的俯仰和侧倾, 崎岖地型中吊索倾斜, 对月球车产生水平干扰力。传统的单索补偿方法是 通过一根连接于车厢的吊索向星球车施加补偿力。 此方法在履带式、无悬架式和弹簧悬架式星球车上 有较多应用, 俄罗斯对上述类型星球车进行了单索 重力补偿试验; 在针对三节式微型火星探测车的试 验中，使用了两层杜杆分配补偿力以避免使用多根 拉力索 ${ }^{[4]}$ 。传统单索补偿在摇臂转向架式星球车的 应用存在两个问题: (1) 不能补偿摇臂构件相对转点 的转矩; (2) 补偿吊索的理想连接点位于车厢内部, 工程实现困难。

为简单、准确地进行低重力模拟, 本文解决限 制单索重力补偿方法应用于摇臂转向架式星球车的 一系列问题。星球车轮压计算是星球车设计、分析 和仿真中的重要内容 ${ }^{[7-9]}$, 针对本文分析的需要, 在 第 1 节建立崎岖地型中轮地接触力的模型, 证明 “正确补偿接触力” 和 “正确补偿狭义接触力” 等 价, 以简化下文证明过程。针对最简单的四轮星球 车, 第 2 节给出使用单根吊索和摇臂配重模拟轮地 接触力的方法(单索-配重方法), 通过证明补偿后的 狭义接触力和低重力环境一致以证明轮地接触力模 拟正确。第 3 节给出单索-配重方法在六轮星球车上 的应用, 解释四、六轮星球车的悬架差别对配重大 小、位置的影响; 由于八轮星球车 ${ }^{[10]}$ 构型与四、六 轮星球车十分相似, 因此其补偿方法不再赘述。第 4 节使用仿真证明单索-配重方法的正确性, 证明在 模拟低于 $g / 6$ 重力环境时此方法相对传统单索方法
具有优势。第 5 节提出相似悬挂原理, 解决补偿吊 索无法连接于车厢封闭体内部的问题。为了避免质 心测量的复杂过程, 第 6 节提出使用星球车在各姿 态下的狭义接触力计算和校正补偿力系的方法, 并 相应给出狭力接触力测量原理和测量平台结构。第 7 节介绍了以上研究的实践应用和经验。第 8 节是 结论。

\section{1 轮地接触力模型}

\section{1 模型定义}

本小节定义轮地接触力模型的坐标系和符号。 建立右手坐标系 $O x y z$ : $y$ 轴正向与重力方向相反, $x$ 轴平行于水平面且使得 $O x y$ 平面与星球车前进方 向平行; 各坐标轴单位矢量为 $\boldsymbol{i}, \boldsymbol{j}, \boldsymbol{k}$ 。定义序号: 星球车副摇臂为前端, 记右前、右中、右后、左前、 左中和左后轮分别为 $1 \sim 6$ 号轮; 记右副摇臂、右前 和右中轮为 1 号构件，右主摇臂和右后轮为 2 号构 件，车厢为 3 号构件，左副摇臂、左前和左中轮为 4 号构件，左主摇臂和后轮为 5 号构件。定义位置: 记右侧主副摇臂铰接点为 $A$, 左侧对应点为 $C$, 右 侧主摇臂与车厢的差速轴连接点为 $B$, 左侧对应点 为 $D$, 差速轴中点为 $E, \quad 1 \sim 5$ 号构件质心位置点分 别为点 $F \sim J, 1 \sim 6$ 号轮与地面接触点记为 $K \sim P$ 。 定义矢量: 记 $N_{k}$ 为接触面对 $k$ 号轮的接触力矢量, 它与各轴投影值关系记为 $\boldsymbol{N}_{k}=H_{k} \boldsymbol{i}+V_{k} \boldsymbol{j}+I_{k} \boldsymbol{k}$; 记 $\boldsymbol{r}_{A B}$ 为从点 $A$ 到点 $B$ 的位置矢量, 它与各轴投影值 的关系记为 $\boldsymbol{r}_{A B}=x_{A B} \boldsymbol{i}+y_{A B} \boldsymbol{j}+z_{A B} \boldsymbol{k}$; 记 $T_{A}\left(N_{1}\right)=N_{1} \times r_{A K}$ 为 $K$ 点接触力 $N_{1}$ 相对 $A$ 点的矩 矢, 简记为 $\boldsymbol{T}_{N 1}^{A}$, 它与各轴投影值关系记为 $\boldsymbol{T}_{N k}^{\boldsymbol{A}}=X_{N k}^{A} \boldsymbol{i}+Y_{N k}^{A} \boldsymbol{j}+Z_{N k}^{A} \boldsymbol{k} ;$ 记 $m_{k}$ 和 $\boldsymbol{G}_{k}$ 为 $k$ 号构件的 质量和重力。其余定义: 记车侧倾角为 $\alpha$, 记模拟 低重力加速度值为 $g / s, s>1$ 。图 $1 \mathrm{a}$ 是 $O x y$ 平面视图, 图 $1 \mathrm{~b}$ 是 $O y z$ 平面视图。

为了表达摇臂旋转关节的转矩传递特性，将坐 标系 $O x y z$ 绕 $x$ 轴旋转 $\alpha$ 角, 得到新坐标系 $O x^{\prime} y^{\prime} z^{\prime}$, 使 $O x^{\prime} y^{\prime}$ 平面与摇臂转动关节的轴线垂直, 如图 $1 \mathrm{~b}$ 所示。将 $O x^{\prime} y^{\prime}$ 称为俯仰平面、 $O y^{\prime} z^{\prime}$ 称为侧倾平面、 $O z^{\prime} x^{\prime}$ 称为转向平面。 $N_{1}$ 相对 $A$ 点矢矩 $\boldsymbol{T}_{N 1}^{A}$ 在坐标 系 $O x^{\prime} y^{\prime} z^{\prime}$ 各轴投影值 $X_{N 1}^{A}, Y_{N 1}^{A}$ 和 $Z_{N 1}^{A}$ 和在 $O x y z$ 各 轴投影值 $X_{N 1}^{A}, Y_{N 1}^{A}$ 和 $Z_{N 1}^{A}$ 的关系为

$$
\left(\begin{array}{l}
X_{N 1}^{A}{ }^{\prime} \\
Y_{N 1}^{A}{ }^{\prime} \\
Z_{N 1}^{A}{ }^{\prime}
\end{array}\right)=\left(\begin{array}{ccc}
1 & 0 & 0 \\
0 & \cos \alpha & -\sin \alpha \\
0 & \sin \alpha & \cos \alpha
\end{array}\right)\left(\begin{array}{l}
X_{N 1}^{A} \\
Y_{N 1}^{A} \\
Z_{N 1}^{A}
\end{array}\right)
$$




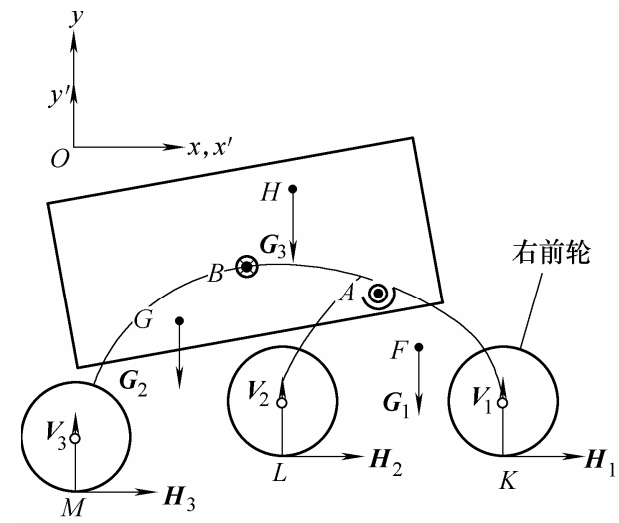

(a) 正视图

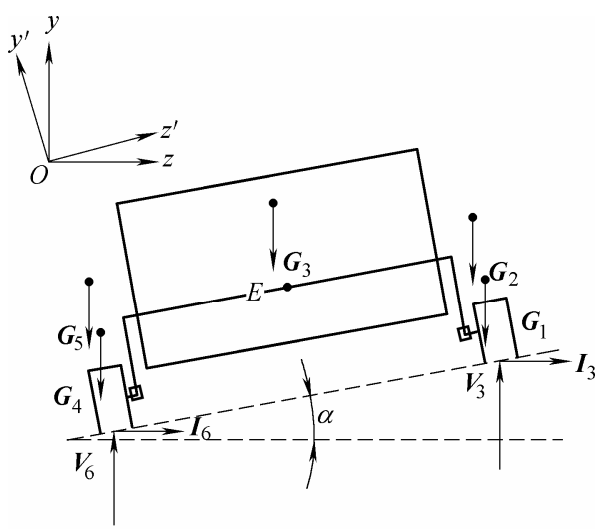

(b) 侧视图

图 1 六轮星球车的坐标和符号定义

\section{2 星球车轮地接触力模型}

本节建立轮地接触力的准静态模型。首先分析 转矩平衡关系。在 $O x^{\prime} y^{\prime}$ 平面内副摇臂转动关节不传 递转矩。相对 $z^{\prime}$ 轴, 构件 1 上接触力 $N_{1} 、 N_{2}$ 和重力 $G_{1}$ 相对 $A$ 点的转矩投影 $Z_{N 1}^{A \prime} 、 Z_{N 2}^{A}{ }^{\prime}$ 和 $Z_{G 1}^{A}$ 互相平衡, 如图 2a 所示, 即

$$
Z_{N 1}^{A{ }^{\prime}}+Z_{N 2}^{A}{ }^{\prime}=-Z_{G 1}^{A \prime}
$$

$O x^{\prime} y^{\prime}$ 平面内, 差速器传递给单侧摇臂的转矩 $T_{B}$ 是车厢质心相对差速轴转矩的 $1 / 2$, 即 $T_{B}=Z_{G 3}^{B}{ }^{\prime} / 2$ 。相对 $z^{\prime}$ 轴, 构件 2 上接触力 $N_{3} 、 A$ 点内力 $-N_{A}$ 和重力 $\boldsymbol{G}_{2}$ 相对 $B$ 点转矩 $Z_{N 3}^{B}{ }^{\prime} 、 Z_{-N A}^{B}{ }^{\prime}$ 和 $Z_{G 2}^{B \prime}$ 平衡了差速器传至构件 2 的转矩 $T_{B}$, 如图 $2 \mathrm{~b}$ 所示。构件 2 转矩平衡式为

$$
Z_{N 3}^{B}{ }^{\prime}+Z_{-N A}^{B}{ }^{\prime}=-Z_{G 2}^{B}{ }^{\prime}-Z_{G 3}^{B{ }^{\prime}} / 2
$$

构件 4、5 转矩平衡方程与式(2)、(3)相似, 分 别是

$$
\begin{gathered}
Z_{N 4}^{C}{ }^{\prime}+Z_{N 5}^{C}{ }^{\prime}=-Z_{G 4}^{C}{ }^{\prime} \\
Z_{N 6}^{D}{ }^{\prime}+Z_{-N C}^{D}{ }^{\prime}=-Z_{G 5}^{D}{ }^{\prime}-Z_{G 3}^{D \prime} / 2
\end{gathered}
$$

在 $O y^{\prime} z^{\prime}$ 平面内星球车没有自由度。由于 $x^{\prime}$ 轴 与 $x$ 轴重合, 轮地接触力 $x$ 轴分量 $H$ 相对 $x^{\prime}$ 轴无转 矩。相对 $x^{\prime}$ 轴, 轮地接触力坚直分量 $V$ 、水平分量

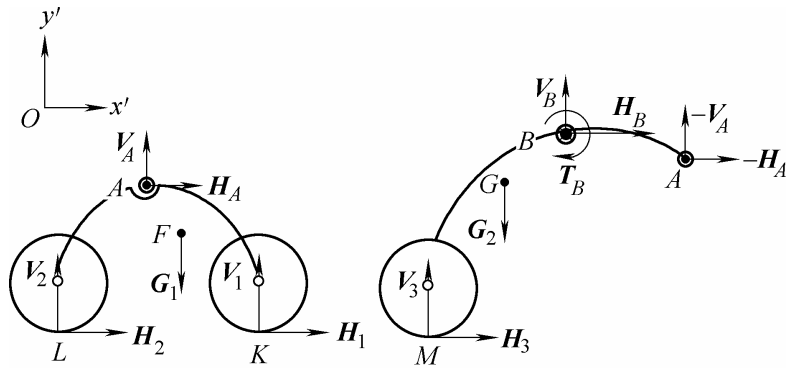

(a) 副摇臂构件受力分析

(b) 主摇臂构件受力分析

图 2 摇臂构件的分离体受力分析

$I$ 和质心重力 $G$ 相对任意点(可选为差速轴中点 $E$ ) 的转矩互相平衡, 如图 $1 \mathrm{~b}$ 所示。平衡关系记为

$$
\sum\left(X_{V}^{E^{\prime}}+X_{I}^{E^{\prime}}\right)=-\sum X_{G}^{E^{\prime}}
$$

在 $O z^{\prime} x^{\prime}$ 平面星球车没有自由度。相对 $y^{\prime}$ 轴, 轮地接触力的三向分量 $H 、 V 、 I$ 和重力 $G$ 相对任 意点(可选为差速轴中点 $E$ ) 的转矩互相平衡

$$
\sum\left(Y_{H}^{E^{\prime}}+Y_{V}^{E^{\prime}}+Y_{I}^{E^{\prime}}\right)=-\sum Y_{G}^{E^{\prime}}
$$

然后分析力平衡。由于在 $x, y, z$ 方向上整车受 力平衡, 因此

$$
\sum H=0 \quad \sum V=-\sum G \quad \sum I=0
$$

联立式(2) (8), 将式(1)代入式(2) (7), 获得 转矩平衡关系在坐标系 $O x y z$ 内的表达, 将构件 1、 3 的力平衡关系代入式(3)、(5)以消除内力项 $Z_{-N A}^{B}{ }^{\prime}$ 、 $Z_{-N C}^{B}{ }^{\prime}$ 得到轮地接触力平衡模型

$$
\left\{\begin{array}{l}
\sum H=0 \quad \sum V=-\sum G \quad \sum I=0 \\
\sum\left(X_{V}^{E}+X_{I}^{E}\right)=-\sum X_{G}^{E} \\
\sum\left[\left(Y_{H}^{E}+Y_{I}^{E}\right)-\tan \alpha\left(Z_{H}^{E}+Z_{V}^{E}\right)\right]=\sum \tan \alpha Z_{G}^{E} \\
\tan \alpha\left(Y_{N 1}^{A}+Y_{N 2}^{A}\right)+\left(Z_{N 1}^{A}+Z_{N 2}^{A}\right)=-\tan \alpha Y_{G 1}^{A}-Z_{G 1}^{A} \\
\tan \alpha\left(Y_{N 4}^{C}+Y_{N 5}^{C}\right)+\left(Z_{N 4}^{C}+Z_{N 5}^{C}\right)=-\tan \alpha Y_{G 4}^{C}-Z_{G 4}^{C} \\
\tan \alpha\left[Y_{N 3}^{B}+z_{B A}\left(H_{1}+H_{2}\right)-x_{B A}\left(I_{1}+I_{2}\right)\right]+\left[Z_{N 3}^{B}+\right. \\
\left.\quad x_{B A}\left(V_{1}+V_{2}\right)-y_{B A}\left(H_{1}+H_{2}\right)\right]=-\left(x_{B A} G+Z_{G 2}^{B}+Z_{G 3}^{E} / 2\right) \\
\tan \alpha\left[Y_{N 6}^{D}+z_{D C}\left(H_{4}+H_{5}\right)-x_{D C}\left(I_{4}+I_{5}\right)\right]+\left[Z_{N 6}^{D}+\right. \\
\left.\quad x_{D C}\left(V_{4}+V_{5}\right)-y_{D C}\left(H_{4}+H_{5}\right)\right]=-\left(x_{D C} G_{4}+Z_{G 5}^{D}+Z_{G 3}^{E} / 2\right)
\end{array}\right.
$$

只将无法简写的转矩写为力和力臂乘积形式。因为 模型式(9)的等号左边只有含接触力的项及其系数, 等号右边只有含质心重力的项及其系数, 所以轮地 接触力模型是轮地接触力系和质心重力系之间的线 性映射。将接触力模型写为矢量方程形式

$$
\boldsymbol{R}_{9 \times 18} \boldsymbol{n}_{18 \times 1}=\boldsymbol{b}_{9 \times 1} g
$$

式中, $\boldsymbol{n}_{18 \times 1}=\left(H_{1}, H_{2}, \cdots, H_{6}, V_{1}, V_{2}, \cdots, V_{6}, I_{1}, I_{2}, \cdots, I_{6}\right)$ 是接触力系投影矢量, $\boldsymbol{b}_{9 \times 1} g$ 由等号右边的质心重力 项及其系数构成。系数矩阵 $\boldsymbol{R}_{9 \times 18}$ 由星球车姿态唯一 
确定, 它的前三行为力平衡方程的系数, 元素为 1 、 -1 或 0 ; 后六行由转矩平衡方程的系数构成, 是力 臂矢径在 $x, y, z$ 的投影。方程组式(9)由线性无 关的平衡方程构成, 因此系数阵秩 $R(\boldsymbol{R})$ 与增广阵秩 $R(\boldsymbol{R}, \boldsymbol{b})$ 同为 9 , 解集秩为 $18-9=9$ 。接触力解的结 构为

$$
\boldsymbol{n}=\boldsymbol{\eta}+\sum_{i=1 \sim 9} c_{i} \boldsymbol{\xi}_{i}
$$

式中, $\boldsymbol{\xi}_{i}, i=1 \sim 9$ 是齐次方程 $\boldsymbol{R}_{9 \times 18} \boldsymbol{n}_{18 \times 1}=\boldsymbol{O}$ 的基础解 系, $c_{i}$ 是任意实数, $\boldsymbol{\eta}$ 是方程式(10)的特解。

接触力模型欠约束的原因是重力补偿不需要 考虑各轮转速关系和沙土性质的约束。使用接触力 模型讨论重力补偿过于繁琐, 下节给出更简练的 方法。

\section{3 狭义接触力及其性质}

定义: 狭义接触力是所有轮地接触力水平分量 都为零时的特例。首先证明狭义接触力的唯一存在 性。将形为 $\boldsymbol{n}_{18 \times 1}=\left(\boldsymbol{0}_{6 \times 1}, V_{1}, V_{2}, \cdots, V_{6}, \boldsymbol{0}_{6 \times 1}\right)$ 的狭义接触 力矢量代入方程组式(9), 使其退化为狭义接触力 模型

$$
\left\{\begin{array}{l}
\sum V=-\sum G \\
\sum X_{V}^{E}=-\sum X_{G}^{E} \\
Z_{N 1}^{A}+Z_{N 2}^{A}=-Z_{G 1}^{A} \\
Z_{N 4}^{C}+Z_{N 5}^{C}=-Z_{G 4}^{C} \\
Z_{N 3}^{B}+x_{B A}\left(V_{1}+V_{2}\right)=-\left(x_{B A} G+Z_{G 2}^{B}+Z_{G 3}^{E} / 2\right) \\
Z_{N 6}^{D}+x_{D C}\left(V_{4}+V_{5}\right)=-\left(x_{D C} G_{4}+Z_{G 5}^{D}+Z_{G 3}^{E} / 2\right)
\end{array}\right.
$$

方程组式(12)中线性无关方程个数与未知数个数都 为 6 , 所以狭义接触力唯一存在。即解系式(11)中唯 一存在系数组合 $k_{i}, i=1 \sim 9$, 使得

$$
\left(\boldsymbol{0}_{6 \times 1}, V_{1}, V_{2}, \cdots, V_{6}, \boldsymbol{0}_{6 \times 1}\right)=\boldsymbol{\eta}+\sum_{i=1 \sim 9} k_{i} \boldsymbol{\xi}_{i}
$$

下面证明 “狭义接触力被正确补偿” 和 “接触 力被正确补偿” 等价。假设在地球重力场 $g$ 中, 狭 义接触力被正确补偿为低重力场 $g / s$ 中的数值, 即

$$
\boldsymbol{R}\left(\boldsymbol{\eta}+\sum_{i=1 \sim 9} k_{i} \boldsymbol{\xi}_{i}\right)=\boldsymbol{b} g / s
$$

由于 $\xi_{i}$ 是 $\boldsymbol{R}_{9 \times 18} N_{18 \times 1}=0$ 的基础解系, 因此式(13) 的成立与系数 $k_{i}, i=1 \sim 9$ 取值无关, 即整个接触力解 系式(11)都被正确补偿。因此, “狭义接触力被正确 补偿就是接触力被正确补偿” 命题为真。由于上述 命题的逆命题明显成立, 因而 “狭义接触力被正确 补偿” 和 “接触力被正确补偿” 等价关系得证。

狭义接触力的具有良好性质: (1) 唯一存在;

(2) 其模型在坐标系 $O x^{\prime} y^{\prime} z^{\prime}$ 和坐标系 $O x y z$ 内的表达

一致; (3) 在低重力模拟的讨论中与接触力等价。由
于这些性质, 下文的讨论可以针对狭义接触力简练 地进行。称狭义接触力为轮压, $N_{k}$ 专指 $k$ 号轮的 轮压。

\section{4 轮压补偿正确的判据}

在 $g / s$ 重力场中, 轮压方程为

$$
\boldsymbol{R n}=\boldsymbol{b} g / s
$$

补偿状态下, 补偿力 $\boldsymbol{F}$ 影响构件的力和力矩平衡, 因此轮压方程中包含了补偿力项

$$
\boldsymbol{R} \boldsymbol{n}=\boldsymbol{b} g+g(\boldsymbol{F})
$$

式中, $g(\boldsymbol{F})$ 是补偿力项。因为补偿力和重力都作用 于构件上, 它们的区别仅是作用点和方向不同, 所 以补偿力项 $g(\boldsymbol{F})$ 和重力项 $\boldsymbol{b} g$ 的形式相似。

补偿状态和低重力状态的轮压方程的等号左 边相等。若补偿正确, 两方程等号右边必相等, 即 $\boldsymbol{b} g+g(\boldsymbol{F})=\boldsymbol{b} g / s$, 移项后为

$$
g(\boldsymbol{F})=(1 / s-1) \boldsymbol{b} g
$$

式(14)即为补偿判据。

\section{2 四轮星球车单索-配重补偿方法}

\section{1 补偿力系施加规则}

为证明单索-配重方法对摇臂转向架式星球车 的适用性, 本文分别针对四轮、六轮星球车证明方 法正确。

定义右前、右后、左前和左后车轮为 $1 \sim 4$ 号 轮; 记右摇臂构件、车厢构件和左摇臂构件分别为 $1 \sim 3$ 号构件。记右摇臂与差速轴连接点为 $A$ 、左侧 对应点为 $B$ 、差速轴中点为 $C 、 1 \sim 3$ 号构件质心点 为 $D 、 E$ 和 $F$ 。记作用于 $i$ 号构件的补偿力为 $F_{i}$ 。其 余标记沿用上文规定。下面给出四轮星球车补偿力 系的施加规则。

(1) 在右摇臂构件的 $D^{\prime}$ 点设置配重, 使点 $A$ 位 于线段 $D^{\prime} D$ 上, 见图 $3 \mathrm{a}$ 。使配重重力 $F_{1}$ 、构件自重 $G_{1}$ 相对 $A$ 点的矢矩比值为 $(1 / s-1)$

$$
F_{1} r_{A D^{\prime}}=(1 / s-1) G_{1} r_{A D}
$$

由于点 $D^{\prime} 、 A$ 和 $D$ 共线, 所以不管右摇臂处于何种 姿态, 空间矢量 $\boldsymbol{r}_{A D^{\prime}} 、 \boldsymbol{r}_{A D}$ 在各轴投影的比值恒为 $1 / s-1$, 因而式(15)与下面方程组等价

$$
\left\{\begin{array}{l}
X_{F 1}^{A}=(1 / s-1) X_{G 1}^{A} \\
Y_{F 1}^{A}=(1 / s-1) Y_{G 1}^{A} \\
Z_{F 1}^{A}=(1 / s-1) Z_{G 1}^{A}
\end{array}\right.
$$

引用式(15)时, 总是指使用其等价方程组中的某一 项。这种等价关系在下文定义其他配重时不再重复 给出。

（2）按照相同的方法在左摇臂构件配置配重 
$F_{3}$ 。保证两侧配重重力 $F_{1} 、 F_{3}$ 比值等于构件重力 $G_{1} 、 G_{3}$ 比值

$$
F_{1} / F_{3}=G_{1} / G_{3}=\left(F_{1}+G_{1}\right) /\left(F_{3}+G_{3}\right)
$$

(3) 在车厢构件的质心 $E$ 处施加向上补偿力 $F_{2}$, 见图 $3 \mathrm{~b}$, 使其与车厢重力 $G_{2}$ 比值为 $(1 / s-1)$

$$
F_{2}=(1 / s-1) G_{2}
$$

在差速轴线上寻找特殊点 $K$ 点, 使其满足约束

$$
r_{K A}\left(F_{1}+G_{1}\right)+r_{K B}\left(F_{3}+G_{3}\right)=0
$$

在 $K$ 点施加向上补偿力 $F_{2}{ }^{\prime}$, 见图 3b。其大小为

$$
F_{2}^{\prime}=-\left(F_{1}+F_{3}\right)+(1 / s-1)\left(G_{1}+G_{3}\right)
$$

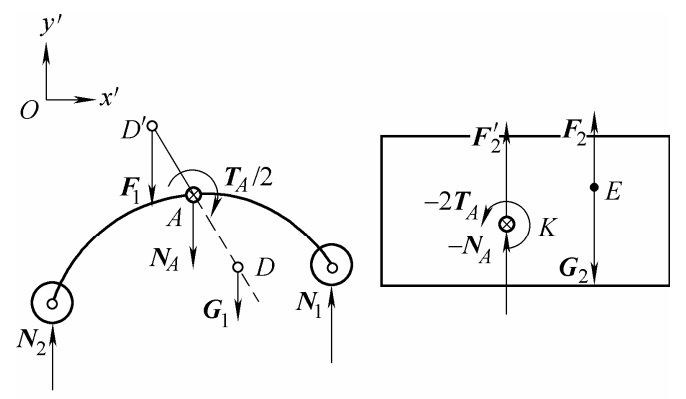

(a) 摇臂配重的施加 $\quad$ (b) 车厢补偿力的施加

图 3 四轮星球车补偿力系的施加

由于 $F_{2}$ 和 $F_{2}{ }^{\prime}$ 在车厢作用点位置、大小不变, 方向始终坚直向上, 因此可将两力合并。合并力的 作用点即为补偿吊索的作用点, 力大小为吊索施加 给星球车的补偿力, 因此实际中只使用一根吊索。 此处使用两个车厢补偿力进行分析以便于理解。

\section{2 轮压模拟效果}

下面证明补偿力系能够在所有姿态下正确补 偿四轮星球车所有轮的轮压。在地球重力环境中, 四轮星球车轮压模型为

$$
\left\{\begin{array}{l}
\sum N=-\sum G \\
\sum X_{N}^{K}=-\sum X_{G}^{K} \\
Z_{N 1}^{A}+Z_{N 2}^{A}=-\left(Z_{G 1}^{A}+Z_{G 2}^{C} / 2\right) \\
Z_{N 3}^{B}+Z_{N 4}^{B}=-\left(Z_{G 3}^{B}+Z_{G 2}^{C} / 2\right)
\end{array}\right.
$$

根据补偿判据, 补偿力和重力的关系应满足

$$
\left\{\begin{array}{l}
F_{1}+F_{2}+F_{2}^{\prime}+F_{3}=(1 / s-1) \sum G \\
X_{F 1}^{K}+X_{F 2}^{K}+X_{F 2^{\prime}}^{K}+X_{F 3}^{K}=(1 / s-1) \sum X_{G}^{K} \\
Z_{F 1}^{A}+\left(Z_{F 2^{\prime}}^{C}+Z_{F 2}^{C}\right) / 2=(1 / s-1)\left(Z_{G 1}^{A}+Z_{G 2}^{C} / 2\right) \\
Z_{F 3}^{B}+\left(Z_{F 2^{\prime}}^{C}+Z_{F 2}^{C}\right) / 2=(1 / s-1)\left(Z_{G 3}^{B}+Z_{G 2}^{C} / 2\right)
\end{array}\right.
$$

将方程组式(20)等号左边的项对应加到轮压模型的 等号左侧, 就是施加补偿力后的轮压模型 $\boldsymbol{R} \boldsymbol{n}-g(\boldsymbol{F})=\boldsymbol{b} g$ 。由于篇幅关系此处不再详细证明。 将式(17)、(19)相加, 并将补偿项移至左边, 重
力项移至右边, 得方程组式(20)中第一式。

由于 $F_{2} 、 G_{2}$ 共点反向, 因此 $Z_{F 2}^{C}=(1 / s-1) Z_{G 2}^{C}$; 由于 $F_{2}{ }^{\prime}$ 在差速轴上、差速轴平行于 $z$ 轴且 $C$ 是差速 轴上一点, 因此 $Z_{F 2^{\prime}}^{C}=0$, 即

$$
Z_{F 2}^{C}+Z_{F 2^{\prime}}^{C}=(1 / s-1) Z_{G 2}^{C}
$$

将式(21)与右摇臂配重约束式(15)相加, 即为方程组 式(20)的第三式。相似地，第四式也成立。

下面证明方程组式(20)中的第二等式成立。将 补偿力和重力关系投影于 $O y^{\prime} z^{\prime}$ 平面, 见图 $4 \mathrm{a}$ 。因 为配重和构件质心是相对于差速轴铰接点 $A 、 B$ 配 置的, 所以计算它们对 $K$ 点转矩时, 总是使用其铰 接点处的等效力系。举例: 为计算 $F_{1}$ 对 $K$ 点转矩, 将其平移至 $A$ 点产生等效力系 $F_{A} 、 T_{A}$, 如图 $4 \mathrm{~b}$ 所 示; 由等效力系的概念可知, 平移前后的力系对任 意点的转矩不变, 即 $X_{F 1}^{K}=T_{A}+F_{A} z_{A K}$ 。

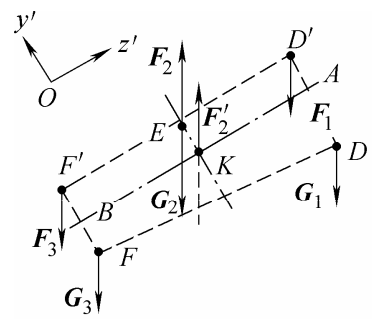

(a) $O y^{\prime} z^{\prime}$ 平面的力系投影

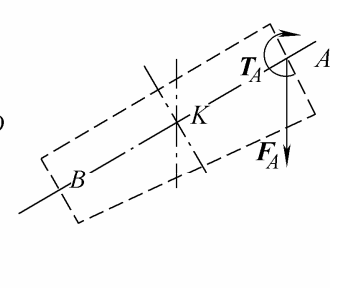

(b) 力的等效平移
图 4 四轮摇臂式星球车受力的 $O y^{\prime} z^{\prime}$ 平面投影

证明分为两部分。

(1) 在 $O y^{\prime} z^{\prime}$ 平面内配重约束式(15)等价于

$$
X_{F 1}^{A}=(1 / s-1) X_{G 1}^{A}
$$

相似地，存在关系

$$
X_{F 3}^{B}=(1 / s-1) X_{G 3}^{B}
$$

(2) 将两侧配重比值的约束式(16)代入式(18)得

$$
\begin{aligned}
& G_{1} z_{K A}+G_{3} z_{K B}=0 \\
& F_{1} z_{K A}+F_{3} z_{K B}=0
\end{aligned}
$$

将式(23) (26)的等号两边相加, 并将等价力系相对 任意点转矩相等的性质代入, 得

$$
X_{F 1}^{K}+X_{F 3}^{K}=(1 / s-1)\left(X_{G 1}^{K}+X_{G 3}^{K}\right)
$$

将关系 $X_{F 2^{\prime}}^{K}=0 、 X_{F 2}^{K}=(1 / s-1) X_{G 2}^{K}$ 与上式相加, 得方程组式(20)第二式。

方程组式(20)成立证毕。在所有姿态下，四轮 星球车全部轮压都被正确补偿至低重力状态。

\section{3 六轮星球车单索-配重补偿方法}

\section{1 补偿力系施加规则}

(1) 在右侧副摇臂构件 $F^{\prime}$ 点设置配重, 令点 $A$ 位于线段 $F F^{\prime}$ 上, 如图 5a 所示。使配重块的重力 $F_{1}$ 
与距离 $r_{A F^{\prime}}$ 满足

$$
F_{1} r_{A F^{\prime}}=(1 / s-1) G_{1} r_{A F}
$$

下面以六轮车为例说明单索重力补偿方法。

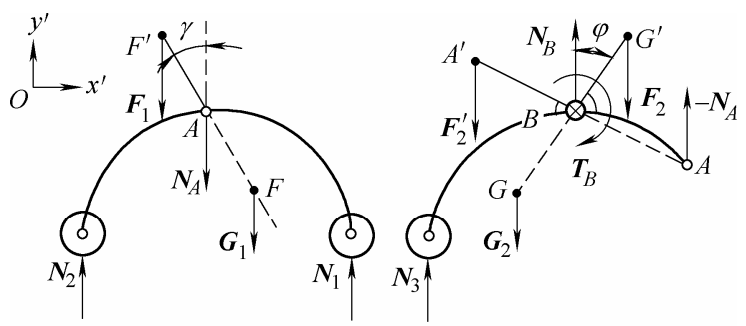

(a) 副摇臂配重的施加 $\quad$ (b) 主摇臂配重的施加

图 5 六轮星球车摇臂配重的施加

(2) 在右侧主摇臂构件的 $G^{\prime}$ 点设置第一配重, 使点 $B$ 位于线段 $G G^{\prime}$ 上, 如图 $5 \mathrm{~b}$ 所示, 使配重块 的重力 $F_{2}$ 与距离 $r_{B G^{\prime}}$ 满足

$$
F_{2} r_{B G^{\prime}}=(1 / s-1) G_{2} r_{B G}
$$

在右侧主摇臂 $A^{\prime}$ 点设置第二配重, 使点 $B$ 位于线段 $A A^{\prime}$ 上, 使配重块的重力 $F_{2}^{\prime}$ 与距离 $r_{B A^{\prime}}$ 满足

$$
F_{2}^{\prime} r_{B A^{\prime}}=(1 / s-1) G_{1} r_{B A}-F_{1} r_{B A}
$$

主摇臂第二配重式(29)中出现了副摇臂构件自重 $G_{1}$ 和配重 $F_{1}$ 。这是因为构件 1 力平衡关系式为

$$
N_{A}=N_{1}+N_{2}+F_{1}+G_{1}
$$

即 $G_{1}$ 和 $F_{1}$ 通过影响 $A$ 点的内力 $-N_{A}$ 而影响主摇臂 构件的转矩平衡。 $G_{1}$ 是星球车自重属性, 因此按照 $(1 / s-1)$ 比例抵消其影响; $F_{1}$ 不是星球车自重属性, 因此按照 -1 比例完全消除其影响。副摇臂构件的重 力和配重影响到主摇臂配重, 这是六轮星球车和四 轮星球车在配重补偿上的主要不同。

（3）左侧副、主摇臂构件上用相同方法配置配 重 $F_{4} 、 F_{5}$ 和 $F_{5}{ }^{\prime}$, 保证右、左两侧配重比值有如下 关系

$$
\frac{F_{1}+F_{2}+F_{2}^{\prime}}{F_{4}+F_{5}+F_{5}{ }^{\prime}}=\frac{G_{1}+G_{2}}{G_{4}+G_{5}}
$$

(4) 在车厢构件的质心 $H$ 处设置补偿力

$$
F_{3}=(1 / s-1) G_{3}
$$

在差速轴线上设置 $Q$ 点, 使得

$$
r_{Q A}\left(G_{1}+G_{2}\right)+r_{Q B}\left(G_{4}+G_{5}\right)=0
$$

在 $Q$ 点施加补偿力 $F_{3}{ }^{\prime}$, 使得

$$
\begin{aligned}
F_{3}^{\prime}= & -\left(F_{1}+F_{2}+F_{2}^{\prime}+F_{4}+F_{5}+F_{5}^{\prime}\right)+ \\
& \left(G_{1}+G_{2}+G_{4}+G_{5}\right)(1 / s-1)
\end{aligned}
$$

\section{2 轮压模拟效果}

根据六轮车轮压方程组式(12)和补偿判据式 (14), 补偿力系和质心力系的关系应满足

$$
\left\{\begin{array}{l}
\sum F=(1 / s-1) \sum G \\
\sum X_{F}^{Q}=(1 / s-1) \sum X_{G}^{Q} \\
Z_{F 1}^{A}=(1 / s-1) Z_{G 1}^{A} \\
Z_{F 4}^{C}=(1 / s-1) Z_{G 4}^{C} \\
Z_{F 2}^{B}+Z_{F 2^{\prime}}^{B}+F_{1} x_{B A}+Z_{F 3}^{E}+Z_{F 3^{\prime}}^{E}= \\
\quad(1 / s-1)\left(x_{B A} G_{1}+Z_{G 2}^{B}+Z_{G 3}^{E} / 2\right) \\
Z_{F 5}^{D}+Z_{F 5^{\prime}}^{D}+F_{4} x_{D C}+Z_{F 3}^{D}+Z_{F 3^{\prime}}^{D}= \\
\quad(1 / s-1)\left(x_{D C} G_{4}+Z_{G 5}^{D}+Z_{G 3}^{E} / 2\right)
\end{array}\right.
$$

方程组式(34)第一式是式(31)和式(33)加和的结果; 第三式是右副摇臂配重式(27)的等价关系; 相似地, 第四式成立。下面证明第二、五、六式成立。

将主摇臂第一配重约束式(28)和第二配重约束 式(29)在 $O y z$ 平面的等价关系相加得

$$
Z_{F 2}^{B}+Z_{F 2^{\prime}}^{B}+F_{1} x_{B A}=(1 / s-1)\left(G_{1} x_{B A}+Z_{G 2}^{B}\right)
$$

由于车厢补偿力 $F_{3}$ 和重力 $G_{3}$ 共点反向, 车厢 补偿力 $F_{3}^{\prime}$ 在差速轴上, 因此

$$
Z_{F 3^{\prime}}^{E}+Z_{F 3}^{E}=(1 / s-1) Z_{G 3}^{E} / 2
$$

将式(35)和式(36)相加即得方程组式(34)第五式。相 似地，第六式成立。

下面证明方程组式(34)中的第二式成立。将补 偿力和重力关系投影于 $O y^{\prime} z^{\prime}$ 平面, 见图 $6 \mathrm{a}$ 。与第 2.2 节方法相似, 仍旧将摇臂质心重力和配重重力移 至 $B 、 D$ 点获得等效力系, 使用等效力系分析它们 对 $Q$ 点的力矩, 如图 6b 所示。

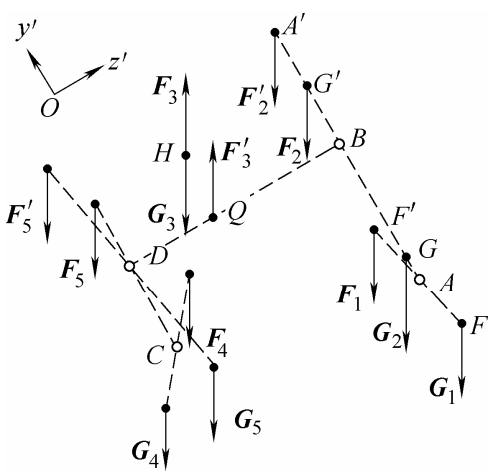

(a) $O y^{\prime} z^{\prime}$ 平面的力系投影

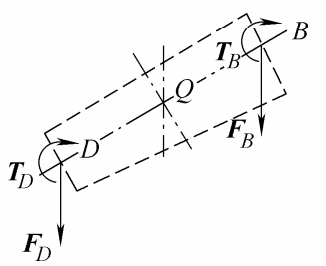

(b) 力的等效平移

图 6 六轮摇臂式星球车受力的 $O y^{\prime} z^{\prime}$ 平面投影 证明分为两部分。

(1) 将 $F_{1} 、 F_{2}$ 和 $F_{2}{ }^{\prime}$ 对 $B$ 点转矩加, 并将 $z_{B F^{\prime}}=z_{B A}+z_{A F^{\prime}}$ 关系代入 


$$
\begin{gathered}
X_{F 1}^{B}+X_{F 2}^{B}+X_{F 2^{\prime}}^{B}= \\
F_{1}\left(z_{B A}+z_{A F^{\prime}}\right)+F_{2} z_{B G^{\prime}}+F_{2}{ }^{\prime} z_{B A^{\prime}}
\end{gathered}
$$

将配重 $F_{1} 、 F_{2}$ 和 $F_{2}{ }^{\prime}$ 的约束式(27) (29)代入式(37)

$$
X_{F 1}^{B}+X_{F 2}^{B}+X_{F 2^{\prime}}^{B}=(1 / s-1)\left(X_{G 1}^{B}+X_{G 2}^{B}\right)
$$

相似于式(38), 有

$$
X_{F 4}^{D}+X_{F 5}^{D}+X_{F 5^{\prime}}^{D}=(1 / s-1)\left(X_{G 4}^{D}+X_{G 5}^{D}\right)
$$

(2) 与第 2.2 节中相似, 将两侧配重比值的约束 式(30)代入 $Q$ 点约束式(32), 得

$$
\begin{gathered}
\left(F_{1}+F_{2}+F_{2}{ }^{\prime}\right) z_{B Q}+\left(F_{4}+F_{5}+F_{5}{ }^{\prime}\right) z_{D Q}=0 \\
0=\left(G_{1}+G_{2}\right) z_{B Q}+\left(G_{4}+G_{5}\right) z_{D Q}
\end{gathered}
$$

将式(38) (41)等号两边分别相加, 并将等价力系相 对任意点转矩相等的性质代入, 得

$$
\sum_{i=1,2,4,5} X_{F i}^{Q}+X_{F 2^{\prime}}^{Q}+X_{F 5^{\prime}}^{Q}=(1 / s-1) \sum_{i=1,2,4,5} X_{G i}^{Q}
$$

将式(42)与 $X_{F 3^{\prime}}^{Q}=0 、 X_{F 3}^{Q}=(1 / s-1) X_{G 3}^{Q}$ 相加, 得方 程组式(34)第二式。

方程组式(34)成立证毕。在所有姿态下, 六轮 星球车的全部轮压都被正确补偿至低重力状态。

\section{3 主摇臂配重化简}

利用车厢通过差速轴向主摇臂传递转矩的特 性化简主摇臂配重。将整车置于水平地面。将主摇 臂的两个配重等效分解为水平、竖直两个方向的配 重。按照质心分解原则, 将 $A^{\prime}$ 点配重分解为 $B$ 点正 上方 $R$ 点和水平方向 $R^{\prime}$ 点两个配重块; 使用同样方 法分解 $G^{\prime}$ 点配重块, 见图 7a。将点 $R^{\prime}$ 和 $S^{\prime}$ 点的配重 合并为新配重, 位置记为 $T^{\prime}$, 将点 $R$ 和 $S$ 点的配重 合并为新配重，位置记为 $T$, 见图 7b。

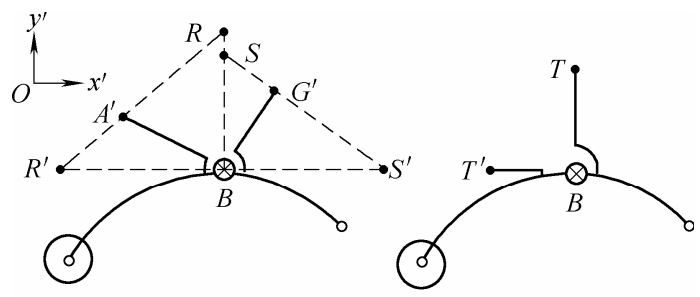

(a) 质心等效分解

(b) 质心等效合并

图 7 主摇臂配重的等效转换

取消 $T^{\prime}$ 点配重, 主摇臂因此损失转矩。为补偿 损失的转矩, 将原作用于差速轴的车厢补偿力 $F_{2}^{\prime}$ 沿 $x$ 轴移动 $\Delta x$, 见图 8 , 使 $F_{2}^{\prime}$ 对差速轴的转矩等于 一侧主摇臂损失转矩的两倍。

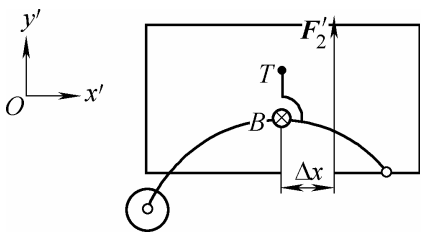

图 8 平移车厢力
主摇臂因取消配重而损失的转矩 $T_{1}$ 和差速器 补偿的转矩的 $T_{2}$ 的关系为

$$
T_{2} / T_{1}=\cos \beta / \cos (\beta+\phi)
$$

式中, $\beta$ 为车厢俯仰角, $\phi$ 为差速器转角。 $\phi=0^{\circ}$ 时, $T_{2} / T_{1}=1$; 普通工况下, 取 $0^{\circ} \leqslant \beta \leqslant 10^{\circ}, 0^{\circ} \leqslant \phi \leqslant 10^{\circ}$, $0.98 \leqslant T_{2} / T_{1} \leqslant 1.05$, 误差率小于 $5 \%$; 极限工况下, 取 $\beta=\phi=20^{\circ}, T_{2} / T_{1}=1.23$, 误差率 $23 \%$ 。注意此 误差率是相对 $T_{1}$ 得出的, 实际中此误差对轮压的影 响可以忽略。

\section{4 单索-配重方法仿真验证}

\section{1 仿真模型及参数}

在动力学仿真软件中构建六轮摇臂星球车模 型。将差速轴中点取为坐标原点。建立右手坐标系, 前进方向取为 $x$ 轴正方向, $y$ 轴坚直向上, 见图 9a。 设置两侧轮中心距 $748 \mathrm{~mm}$, 同侧相邻轮中心距 $585 \mathrm{~mm}$, 车轮半径 $165 \mathrm{~mm}$ 。由于星球车模型关于 Oxy 平面对称, 只给出车厢和一侧悬架的属性, 如 表 1 所示。

表 1 星球车模型质量及尺寸属性

\begin{tabular}{lcccc}
\hline \multicolumn{1}{c}{ 关键位置点 } & $\begin{array}{c}\text { 质量 } \\
m / \mathrm{kg}\end{array}$ & $\begin{array}{c}\text { 坐标 } \\
x / \mathrm{mm}\end{array}$ & $\begin{array}{c}\text { 坐标 } \\
y / \mathrm{mm}\end{array}$ & $\begin{array}{c}\text { 坐标 } \\
z / \mathrm{mm}\end{array}$ \\
\hline 车厢构件质心 & 101 & 100 & 80 & 0 \\
主摇辟构件质心 & 4 & -460 & -320 & 353 \\
副摇譬构件质心 & 5.5 & 338 & -390 & 369 \\
右前轮中心 & - & 585 & -411 & 374 \\
主副摇臂绞接点 & - & 248 & -146 & 334 \\
差速轴中心 & - & 0 & 0 & 0 \\
\hline
\end{tabular}

地面模型设置为刚体。地型分为四段, 见图 9b: 仿真开始时车置于水平段, 然后通过长 $3000 \mathrm{~mm}$ 、 斜度 $28.5^{\circ}$ 下坡段, 然后通过长度 $2500 \mathrm{~mm}$ 水平段, 最后通过深 $150 \mathrm{~mm}$ 长 $3500 \mathrm{~mm}$ 的单侧槽坑段。星 球车在下坡段产生整车俯仰，在侧坑段同时产生整 车俯仰和侧倾, 见图 10a。在开入和开出下坡时, 主副摇臂铰接点产生转角, 在侧坑段主副摇臂铰接 点和差速器同时产生转角, 见图 $10 \mathrm{~b}$ 。

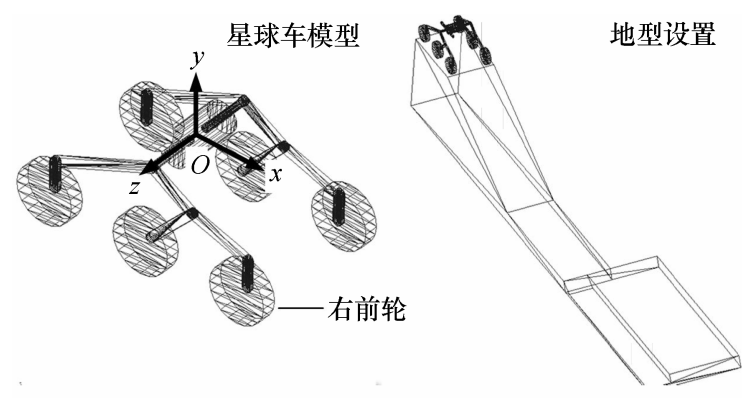

(a) 星球车模型及坐标系

(b) 地面模型

图 9 仿真模型及坐标系 

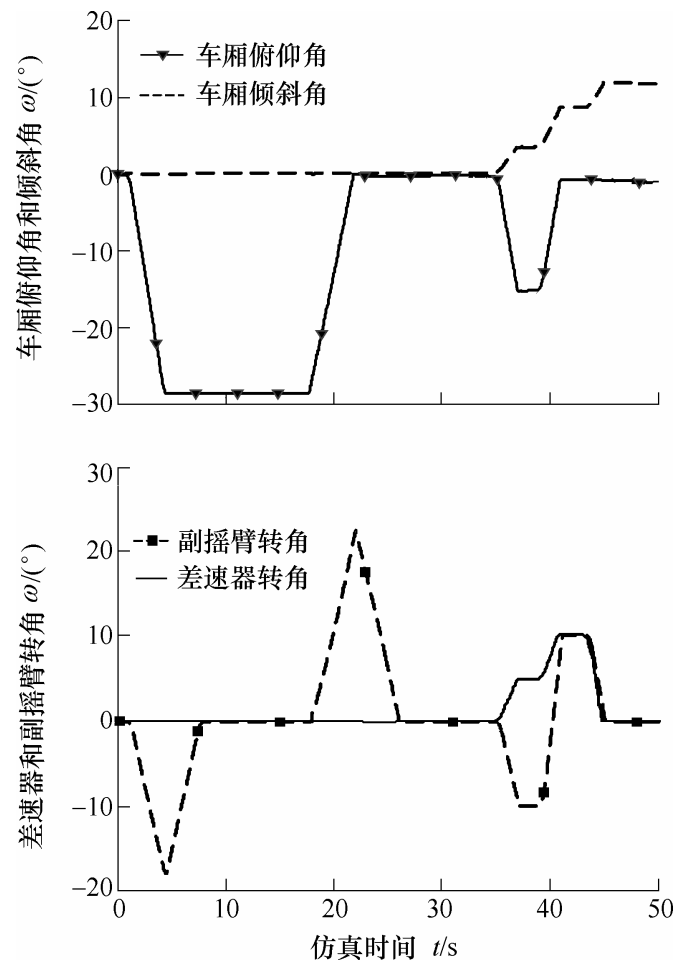

图 10 仿真中悬架姿态的变化

\section{2 仿真结果}

为获得各轮在低重力环境下的标准轮压, 设置 加速度为 $g / s$ 且不施加补偿力, 结果以虚线加方块 表示。后面的仿真中加速度设为 $g$ 。第一、二组仿 真中仅向车厢上的整车合质心位置施加补偿力, 以 模拟传统单索补偿。第一组设置补偿力为整车重力 的 $5 / 6$ 以模拟 $g / 6$ 环境。结果表明: 在通过单侧槽 坑地形时, 右中轮与右后轮的轮压补偿误差率约为 $25 \%$, 两轮误差方向相反, 如图 11 所示。第二组设 置补偿力为整车重力的 $11 / 12$, 以模拟 $g / 12$ 环境。 结果表明: 在通过单侧槽坑地形时, 右中轮、右后 轮的轮压补偿误差率约为 $50 \%$, 两轮误差方向相反; 两轮分别在仿真 $40 \mathrm{~s}$ 和 $20 \mathrm{~s}$ 左右轮压为零, 即车轮 被补偿力拉离地面, 见图 12 。第一、第二组仿真说 明, 传统单索重力补偿方法不能无误差地补偿轮压; 模拟重力场越低, 传统方法的误差越大、适用性越 差。第三组仿真中, 按照第 3.1 节方法计算模拟 $g / 6$ 环境的补偿力系, 并按照第 3.3 节方法降低主摇臂 配重质量。补偿力系的参数列于表 2 。仿真结果表 明: 单索-配重补偿方法可以无误差地模拟低重力状 态下星球车轮压, 化简配重方法带来的误差可以忽 略, 如图 13 所示。

表 2 第三组仿真补偿力系参数

\begin{tabular}{|c|c|c|c|c|}
\hline 右侧构件及车厢补偿力 & $\begin{array}{c}\text { 力 } \\
F / \mathrm{N}\end{array}$ & $\begin{array}{l}\text { 坐标 } \\
x / \mathrm{mm}\end{array}$ & $\begin{array}{l}\text { 坐标 } \\
y / \mathrm{mm}\end{array}$ & $\begin{array}{l}\text { 坐标 } \\
z / \mathrm{mm}\end{array}$ \\
\hline 副摇臂配重 & 44.9 & 158 & -98 & 369 \\
\hline 化简的主摇臂配重 & 46.8 & 0 & 500 & 334 \\
\hline 车厢质心补偿力 & 825.3 & 100 & 80 & 0 \\
\hline 平移的车厢补偿力 & 338.4 & 43.9 & 0 & 0 \\
\hline
\end{tabular}
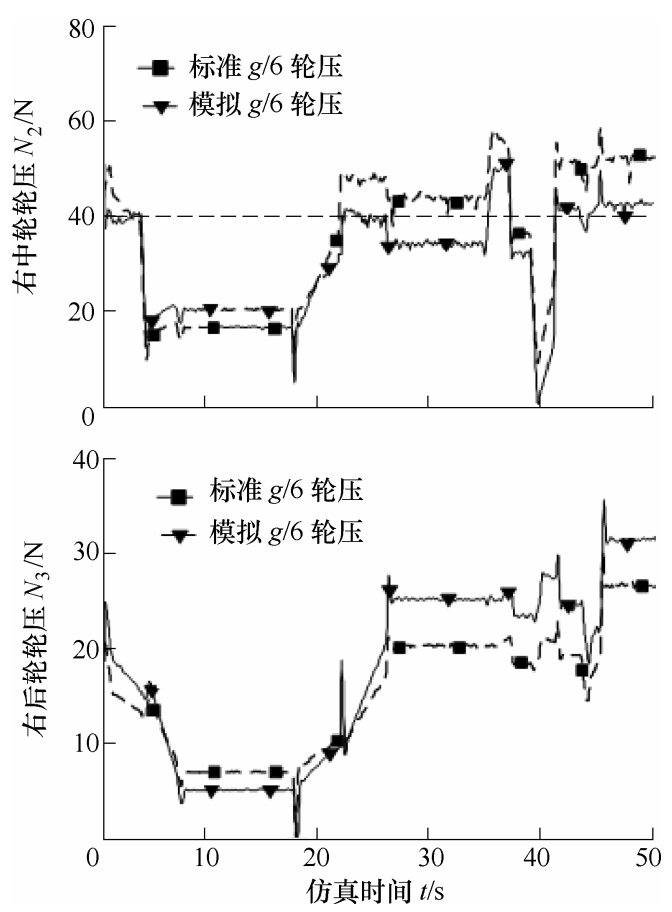

图 11 使用传统单索补偿方法模拟 $g / 6$ 环境轮压
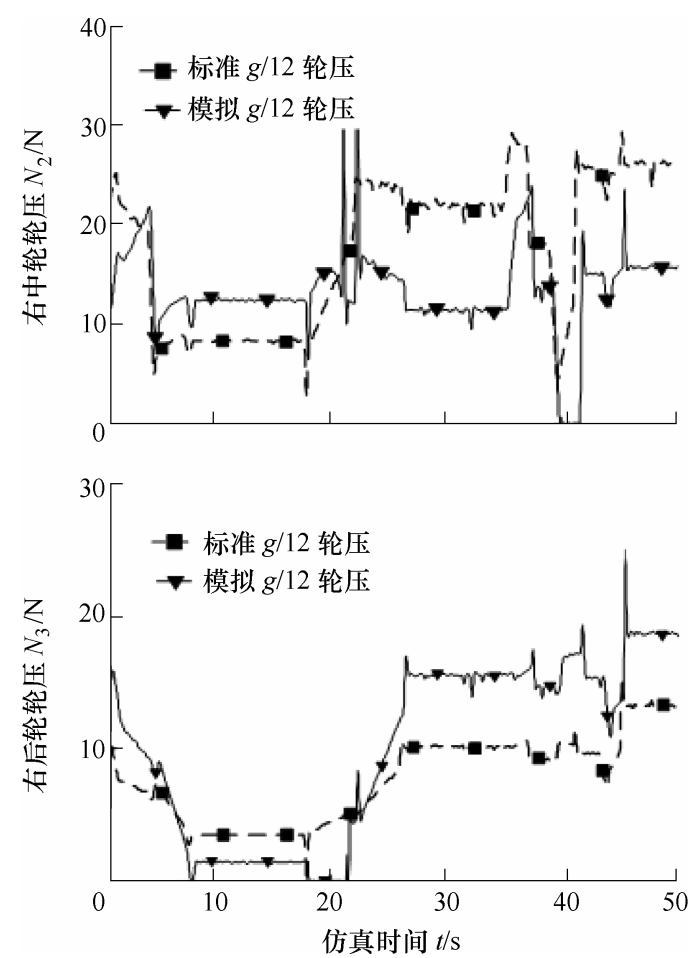

图 12 使用传统单索补偿方法模拟 $g / 12$ 环境轮压

\section{5 相似悬挂原理}

车厢补偿力作用点在车厢内部是星球车重力 补偿的普遍现象。将吊索直接连接于车厢内部有两 个问题: (1) 吊索和车厢内部构件干涉; (2) 在车厢 内部的准确位置预留接口不易实现。本节考虑将补 偿力作用于车厢外部，但使其时时等效作用于车厢 内部固定点。 

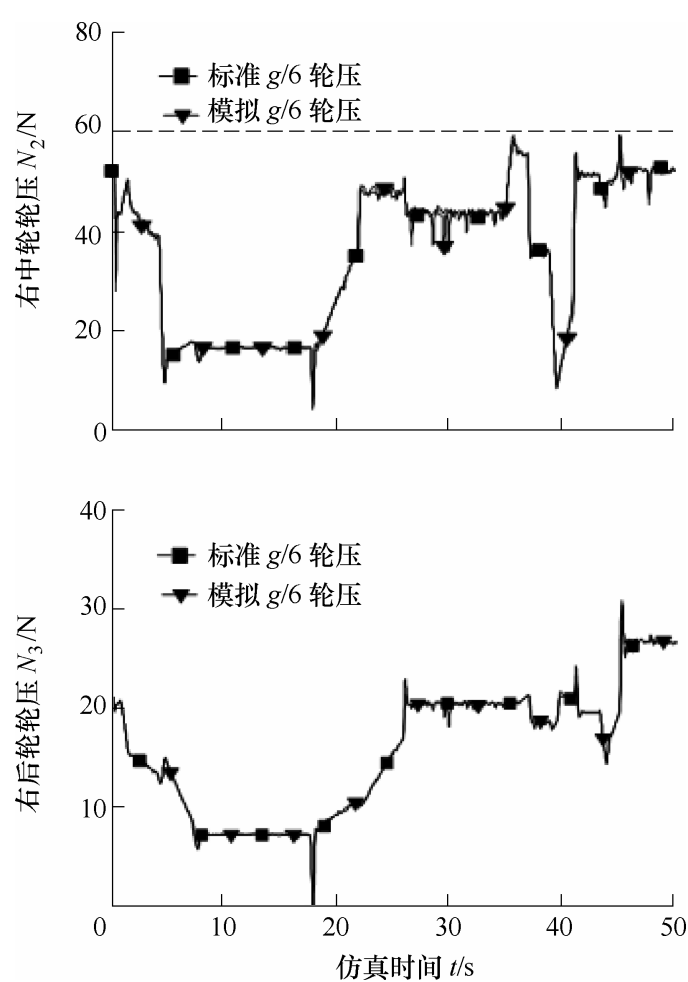

图 13 使用单索-配重补偿方法模拟 $g / 6$ 环境轮压

首先介绍相似悬挂原理。设平面内有两自由物 体, 在两物体上分别取 $\triangle A B C \cong \triangle A^{\prime} B^{\prime} C^{\prime}$, 使用等 长连杆球铰连接 $A A^{\prime} 、 B B^{\prime}$ 以构成平面机构 $A^{\prime} A B B^{\prime}$, 见图 $14 \mathrm{a}$ 。因为 $A^{\prime} A B B^{\prime}$ 和 $A^{\prime} A C C^{\prime}$ 是共用 $A^{\prime} A$ 边的平行四边型, 所以无论机构如何运动, 连 线 $C^{\prime} C$ 始终平行于连杆 $A^{\prime} A$ 。

然后介绍在俯仰平面内向车厢内 $C$ 点施加等效 补偿力的方法。在车厢表面设置球铰连接点 $A 、 B$; 按 $\triangle A B C \cong \triangle A^{\prime} B^{\prime} C^{\prime}$ 关系在悬挂装置上选取球铰 连接点 $A^{\prime} 、 B^{\prime}$ 和吊索连接点 $C^{\prime}$; 使用等长杆连接 $A A^{\prime} 、 B B^{\prime}$, 将吊索连接于 $C^{\prime}$, 在补偿力作用下连 杆 $A^{\prime} A$ 和 $B^{\prime} B$ 始终坚直, 见图 $14 \mathrm{~b}$ 。由于 $C^{\prime} C$ 平行 $A^{\prime} A$, 在俯仰平面内补偿力始终坚直通过星球车内 部点 $C$, 亦即补偿力等效作用于点 $C$ 。

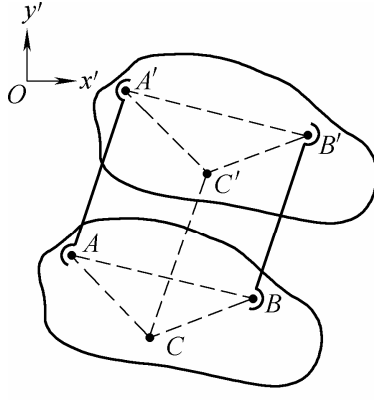

(a) 平面相似悬挂原理

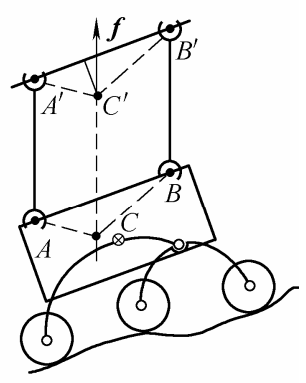

(b) 俯仰面施加等效补偿力
图 14 平面相似悬挂

为使补偿力在空间内等效作用于车厢内部吊 点 $C$, 进行以下构造: 在车厢顶部选四个球铰连接
点 $A 、 B 、 D$ 和 $E$, 与点 $C$ 构成四棱雉 $C A B D E$; 按 $C^{\prime} A^{\prime} B^{\prime} D^{\prime} E^{\prime} \cong C A B D E$ 全等关系在悬挂装置上设置 球铰连接点 $A^{\prime} 、 B^{\prime} 、 D^{\prime} 、 E^{\prime}$ 和吊索连接点 $C^{\prime}$; 使 用等长连杆连接对应点, 将吊索连接于 $C^{\prime}$, 如图 $15 \mathrm{a}$ 所示。图 $15 \mathrm{a}$ 在俯仰平面投影为图 $14 \mathrm{~b}$, 在侧倾 平面的投影为图 $15 \mathrm{~b}$ 。在忽略悬挂装置自重情况下, 整车俯仰、侧倾时吊索补偿力始终等效作用于车厢 内部点。

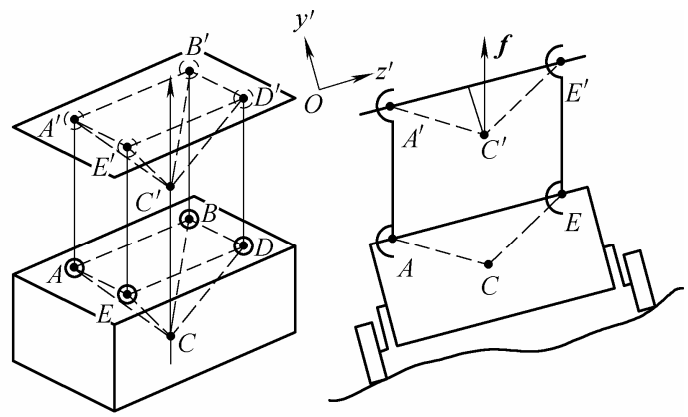

(a) 空间施加等效补偿力

(b) 侧倾平面投影

图 15 空间相似悬挂

为了完全消除悬挂装置自重的影响, 除补偿力 $\boldsymbol{f}$ 外, 还需要在悬挂装置质心处施加与其自重等大 反向的补偿力 $\boldsymbol{f}^{\prime}$ 。由于力 $\boldsymbol{f}$ 和 $\boldsymbol{f}^{\prime}$ 作点、大小、方 向不变，因此可以合成一个作用点、大小、方向不 变的合力。此合力即为考虑挂架自重情况下的补偿 力, 其作用点即为悬挂装置的实际吊索连接点, 其 大小为吊索拉力，因此仍旧是单索补偿。

因为星球车的设计速度普遍较低, 而且球铰关 节提供阻尼, 所以相似悬挂装置的振荡情况可以忽 略。在试验中观测不到震荡。

\section{6 补偿力系的计算和校正方法}

\section{1 配重重量及安装位置计算}

机械系统质心的实际值与设计值往往不同。如 果使用质心信息计算补偿力系, 必须拆解星球车进 行构件的质心测量。此外, 补偿力系的安装缺乏定 位基准, 实际操作中无法确定补偿效果。针对上述 问题，本节以六轮星球车为例介绍通过不同姿态下 的轮压计算、校正补偿力系的方法。

首先介绍俯仰平面内右副摇臂构件配重量和 配重安装角度的计算方法。记其转点至质心的矢量 $\boldsymbol{r}_{A F}$ 在 $O x^{\prime} y^{\prime}$ 平面内投影为 $\boldsymbol{l}_{A F}$, 摇臂水平时 $\boldsymbol{l}_{A F}$ 与坚 直方向夹角为 $\gamma, \gamma$ 即为配重安装角, 见图 $5 \mathrm{a}$ 。维 持其余构件不动, 使构件 1 绕 $A$ 点旋转 $\omega$ 角, 轮压 模型式(12)中的右副摇臂平衡式展开写为

$$
-G_{1} l_{A F} \sin (\gamma+\omega)=N_{1} x_{A K}+N_{2} x_{A L}
$$


式中, $\gamma$ 和 $G_{1} l_{A F}$ 是定值未知量; $\omega$ 是设定旋转角、 $N_{1}$ 和 $N_{2}$ 是可测量轮压、 $x_{A K}$ 和 $x_{A L}$ 可根据设计参数 和旋转角 $\omega$ 算出。记未知量 $G_{1} l_{A F}=x_{1}, \sin \gamma=x_{2}$; 记已知量 $\cos \omega=a_{1}, \sin \omega=a_{2}, Z_{N 1}^{A}+Z_{N 2}^{A}=b$, 平 衡方程式(43)改写为

$$
-a_{1} x_{1} x_{2}-a_{2} x_{1} \sqrt{1-x_{2}^{2}}=b
$$

令 $x_{1} x_{2}=x_{1}^{\prime}, x_{1} \sqrt{1-x_{2}^{2}}=x_{2}^{\prime}$, 将式(44)改写为二元 一次方程形式 $a_{1} x_{1}{ }^{\prime}+a_{2} x_{2}{ }^{\prime}=-b$ 。由于系数 $a_{1}, a_{2}, b$ 随 旋转角 $\omega$ 变化, 令 $\omega$ 取值 $\omega_{1} 、 \omega_{2}$ 以获得二元一次 方程组

$$
\left\{\begin{array}{l}
a_{11} x_{1}^{\prime}+a_{21} x_{2}^{\prime}=-b_{1} \\
a_{12} x_{1}^{\prime}+a_{22} x_{2}^{\prime}=-b_{2}
\end{array}\right.
$$

式(45)有唯一解。通过方程组解可以换算俯仰平面 内右副摇臂构件配重量 $F_{1} l_{A F^{\prime}}$ 和安装角 $\gamma$

$$
\begin{gathered}
F_{1} l_{A F^{\prime}}=(1 / s-1) \sqrt{\left(x_{1}^{\prime 2}+x_{2}^{\prime 2}\right)} \\
\sin \gamma=x_{1}^{\prime} / \sqrt{\left(x_{1}^{\prime 2}+x_{2}^{\prime 2}\right)}
\end{gathered}
$$

下面介绍俯仰平面内右主摇臂构件两个配重 的计算。记主摇臂水平时 $\boldsymbol{l}_{B G}$ 与坚直方向夹角为 $\varphi$, $\varphi$ 即为第一配重安装角, 见图 $5 \mathrm{~b}$ 。保持车厢水平, 使构件 2 绕差速轴旋转 $\omega$ 角, 轮压模型式(12)中主 摇臂平衡式展开写为

$$
\begin{gathered}
N_{3} x_{B M}+\left(N_{1}+N_{2}\right) x_{B A}= \\
-G_{2} l_{B G} \sin (\varphi+\omega)-G_{1} x_{B A}-T_{B}
\end{gathered}
$$

式中, $G_{2} l_{B G} 、 \varphi 、 G_{1}$ 和 $T_{B}$ 是定值未知量; 其余量 是已知量。记未知量 $G_{2} l_{B G}, \sin \varphi, G_{1}, T_{B}$ 分别为 $x_{1}, x_{2}, x_{3}, x_{4}$, 记已知量 $\cos \omega, \sin \omega, x_{B A}$ 为 $a_{1}, a_{2}, a_{3}$, 记 $N_{3} x_{B M}+\left(N_{1}+N_{2}\right) x_{B A}$ 为 $b$, 式(46)改写为

$$
a_{1} x_{1} x_{2}+a_{2} x_{1} \sqrt{1-x_{2}^{2}}+a_{3} x_{3}+x_{4}=-b
$$

式(47)的实质是四元一次方程。记 $x_{1} x_{2}=x_{1}^{\prime}$, $x_{1} \sqrt{1-x_{2}{ }^{2}}=x_{2}{ }^{\prime}, x_{3}=x_{3}{ }^{\prime}, x_{4}=x_{4}{ }^{\prime}$ 。式(47)改写为

$$
a_{1} x_{1}^{\prime}+a_{2} x_{2}^{\prime}+a_{3} x_{3}^{\prime}+x_{4}^{\prime}=-b
$$

由于系数 $a_{1}, a_{2}, a_{2}, b$ 随旋转角 $\omega$ 变化, 令 $\omega$ 取 4 个 不同的值, 获得四元一次方程组 $\boldsymbol{A}_{4 \times 4} \boldsymbol{x}_{4 \times 1}^{\prime}=-\boldsymbol{b}_{4 \times 1}$ 。 明显地, 解矢量 $\boldsymbol{x}_{4 \times 1}{ }^{\prime}$ 唯一存在。换算得第一配重在 俯仰平面内配重量 $F_{2} l_{B G^{\prime}}$ 和安装角 $\varphi$

$$
\begin{gathered}
F_{2} l_{B G^{\prime}}=(1 / s-1) \sqrt{x_{1}^{\prime 2}+x_{2}^{\prime 2}} \\
\sin \varphi=x_{1}^{\prime} / \sqrt{x_{1}^{\prime 2}+x_{2}^{\prime 2}}
\end{gathered}
$$

第二配重在俯仰平面内配重量为

$$
F_{2}^{\prime} l_{B A^{\prime}}=(1 / s-1) x_{3}{ }^{\prime} l_{B A}-F_{1} l_{B A}
$$

式中, $l_{B A}$ 是设计尺寸, $F_{1}$ 是构件 1 的配重重力。 第二配重安装角度是设计角度, 不需要计算。
本节不计算侧倾平面内配重重量和安装位置, 这是因为在侧倾平面内星球车力矩平衡方式与刚体 一致, 通过校正车厢补偿力位置可以完全消除配重 位置误差的影响。

\section{2 车厢补偿力位置校正}

下面介绍校正补偿力位置的方法。不施加任何 补偿力和配重, 测量星球车某状态的轮压, 除以 $s$ 后 即为低重力环境中的标准轮压。

将配重按照第 6.1 节计算结果固定于摇臂。将 车厢补偿力粗略作用于理论位置, 记作用点位置误 差各轴分量为 $\Delta x^{\prime}, \Delta y^{\prime}, \Delta z^{\prime}, i$ 号轮轮压误差 $\Delta_{i}=N_{i}-N_{i}^{\prime}$ 。由于整车总重容易测量, 因此补偿力 大小准确。这说明各轮轮压误差总和为零, 所以

$$
\Delta_{1}+\Delta_{2}+\Delta_{3}=-\left(\Delta_{4}+\Delta_{5}+\Delta_{6}\right)
$$

即一侧压差和与另一侧压差和等大反向, 它们以两 侧轮心距为力臂构成力偶, 此力偶与 $F \Delta z^{\prime}$ 平衡, 所 以 $\Delta z^{\prime}$ 可求

$$
\Delta z^{\prime}=-\left(\Delta_{1}+\Delta_{2}+\Delta_{3}\right) z_{K N} / F
$$

轮压误差总和为零的约束也可以写为

$$
\Delta_{3}+\Delta_{6}=-\left(\Delta_{1}+\Delta_{2}+\Delta_{4}+\Delta_{5}\right)
$$

这说明两侧后轮轮压误差和与其余轮轮压误差和等 大反向。它们力臂 $x_{K A}{ }^{\prime}$ 构成力偶, 此力偶与 $F \Delta x^{\prime}$ 平 衡, 所以

$$
\Delta x^{\prime}=-\left(\Delta_{3}+\Delta_{6}\right) x_{K A} / F
$$

按照算得的 $\Delta x^{\prime}, \Delta z^{\prime}$ 值调整车厢补偿力作用点, 消除 误差 $\Delta x^{\prime}$ 和 $\Delta z^{\prime}$ 。

在水平状态下坚直方向位置误差 $\Delta y^{\prime}$ 对轮压没 有影响, 因而将整车侧倾 $\alpha$, 使得 $\Delta y^{\prime}$ 在水平面内 产生投影 $\Delta y^{\prime} \sin \alpha 。 \Delta y^{\prime}$ 和 $\Delta z^{\prime}$ 计算原理相同

$$
\Delta y^{\prime} \sin \alpha=-\left(\Delta_{1}+\Delta_{2}+\Delta_{3}\right) z_{K N} / F
$$

$\Delta y$ 可求。按照计算结果调整作用点位置, 消除 $\Delta y$ 误 差。车厢补偿力调整完成。

\section{3 轮压测量台结构}

轮压测量台是配合补偿力系的计算和校正工 作设计的专用平台, 其原理简图如图 16 所示。测量 台包含 6 个可升降的轮压测量单元。每个单元具备

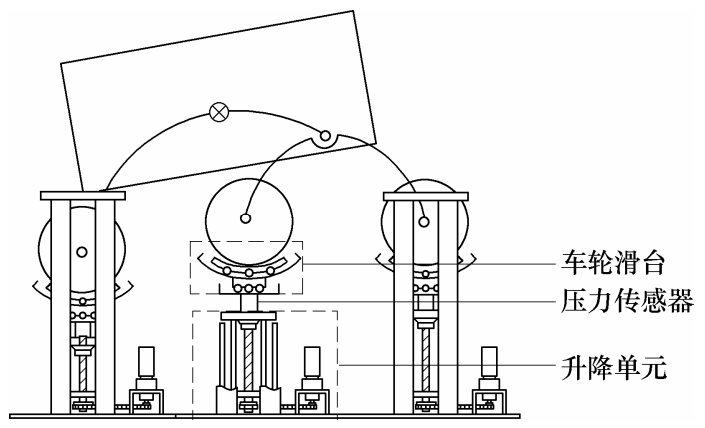

图 16 轮压测量台原理图 
一个压力传感器、一个车轮滑台和一组可单独控制 的升降单元。试验中将星球车置于轮压测量台上, 由升降单元控制各轮相对高度以改变星球车姿态, 由压力传感器读出此姿态下的轮压。

为测量轮压, 必须消除车轮与测量台接触力的 水平分力。以副摇臂为例进行说明轮压测量原理。 将其水平置于轮压测量台上, 车轮和滑台的接触点 用黑点表示, 见图 17a。当副摇臂转动 $\omega$ 角: (1) 前 后轮轴投影距离由 $L$ 变为 $L \cos \omega$; (2) 接触点绕轮 轴转动 $\omega$ 角, 见图 $17 \mathrm{~b}$ 。针对以上现象, 在车轮与 压力传感器之间设计平面滑台和圆弧滑台。使两轮 轴投影距离的变化量被水平滑台吸收, 释放水平分 力; 使接触点绕轮轴的旋转角被圆弧滑台吸收, 释 放接触面对轮轴的转矩。通过压力传感器读出的数 值即为轮压。

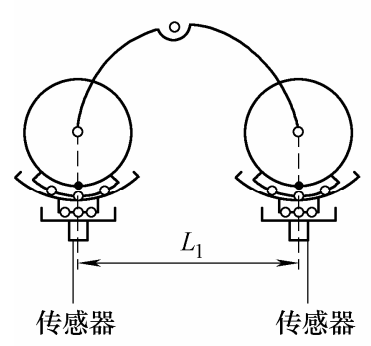

(a) 摇臂最初状态

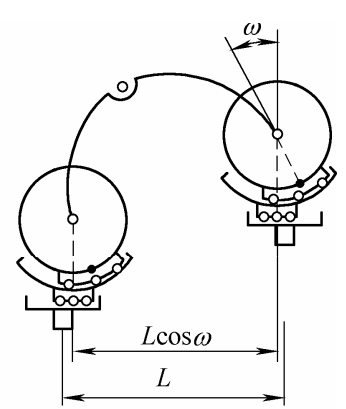

(b) 摇臂旋转后状态
图 17 车轮滑台工作原理

\section{7 研究结果的应用}

利用本文的研究结果, 完成了六轮星球车重力 模拟试验。试验采用了单根吊索和主、副摇臂配重 以模拟六轮星球车在所有姿态下的轮压。为了向车 厢内部等效施加补偿力, 采用了悬挂装置。使用悬 挂装置的吊索连接点微调功能, 可以准确地作用补 偿力, 见图 18 。

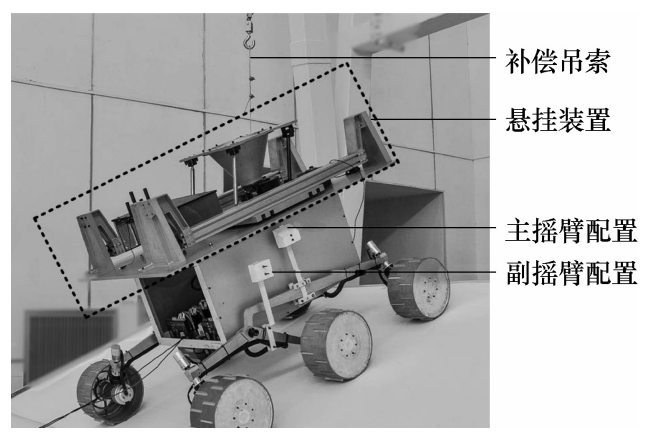

图 18 六轮星球车低重力模拟试验

在模拟月面重力的试验中, 吊索拉力是轮压总 和 7 倍。相应地, 轮压误差总和也是恒拉力系统误
差的 7 倍。这种误差放大效应是重力补偿固有的特 点。为了尽量降低轮压误差、提高系统对星球车坚 直方向冲击的响应速度, 恒拉力系统采用了复杂的 机构和控制策略。若采用多索补偿原理进行模拟试 验, 需要面对很大工程困难。

试验中车厢补偿力的作用点相对车厢顶面的 深度大于车厢高度一半, 直接安装吊索将与内部载 荷干涉。由于误差放大的特点, 吊点位置误差要求 小于 $\pm 2 \mathrm{~mm}$, 车厢内无法预留准确的接口。悬挂装 置的使用解决了以上问题。

轮压测量台(图 19)的使用避免了拆解星球车进 行构件质心测量的繁琐过程, 也为检验补偿效果提 供了依据。由于使用了车轮滑台, 将星球车置于测 量平台之后可以测量多组姿态下的轮压。

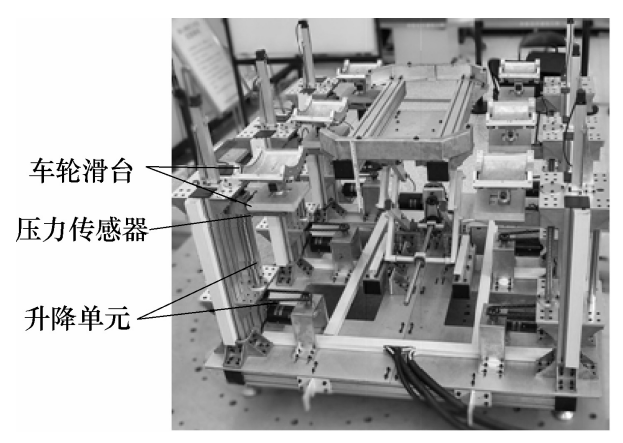

图 19 轮压测量台

实践证明, 本文的研究为摇臂转向架式六轮星 球车的低重力模拟提供了简单、精确、全面的方案 原理和方法。

\section{8 结论}

（1）针对四、六轮星球车建立了崎岖地型中轮 地接触力模型。

（2）证明了狭义接触力具有良好的性质: 它在 接触力模型的解系中唯一存在; “狭义接触力被正确 补偿” 等价于 “接触力被正确补偿”; 狭义接触力模 型在 $O x y z$ 和 $O x^{\prime} y^{\prime} z^{\prime}$ 坐标系内一致。

(3) 以四轮、六轮星球车为例给出单索-配重方 法, 证明了补偿方法正确。

（4）仿真结果表明: 随模拟重力场降低, 传统 单索补偿原理的模拟误差增大; 单索-配重方法 正确。

（5）提出相似悬挂原理, 将作用于悬挂装置的 补偿力始终等效作用于车厢内部理论吊点。

(6) 提出通过星球车不同姿态下的轮压计算、 校正补偿力系的方法, 给出狭义接触力的测量原理, 介绍了轮压测量台的结构。 
(7) 以上研究在六轮星球车重力模拟试验中的 应用取得了良好效果。

\section{参 考 文 献}

[1] KOBAYASHI T, FUJIWARA Y, YAMAKAWA J, et al. Mobility performance of a rigid wheel in low gravity environment[J]. Journal of Terramechanics, 2010, 47(4): 261-274.

[2] KURODA Y, TESHIMA T, SATO Y, et al. Mobility performance evaluation of planetary rover with similarity model experiment[C]// IEEE Robotics \& Automation Society. International Conference on Robotics and Automation, April 26-May 1, 2004, New Orleans, LA. New Orleans, LA: IEEE RAS, 2004: 2098-2103.

[3] VAUGHAN S. Lunar driving simulator history[EB/OL]. [2011-01-10]. www.knology.net/ skeetv/SimHist3.html.

[4] KEMURDJIAN A. Development of simulation means for a gravity forces[C]// Aerospace Division of the ASCE. Robotics 2000, Feb. 27-Mar. 2, 2000, Albuquerque, NM. Albuquerque, NM: ASCE, 2000: 220-225.

[5] RANDEL L, DONALD B, BRIAN $\mathrm{H}$, et al. Mars exploration rover mobility development[J]. IEEE Robotics \& Automation Magazine, 2006, 2(2): 19-26.

[6] WYSOR R B. Preliminary design study of a lunar gravity simulator interim report[R]. New York: NASA, 1967. http : //ntrs.larc.nasa.gov/search.jsp?R=19670021490\&qs= Ns\%3DLoaded-Date\%7C1\%26N\%3D4294842302.

[7] 李所军, 高海波, 邓宗全. 摇臂式探测车质心域计算[J]. 机械工程学报, 2010, 46(17): 8-14.

LI Suojun, GAO Haibo, DENG Zongquan. Calculating domain of center of mass for rocker-bogie exploration rover[J]. Journal of Mechanical Engineering, 2010, 46(17): 8-14.

[8] 马传帅, 文桂林, 周景宇, 等. 月球车沙地行驶动力 学建模与仿真[J]. 机械工程学报, 2011, 47(23): 97-103. MA Chuanshuai, WEN Guilin, ZHOU Jingyu, et al. Whole-vehicle dynamical model and simulation for lunar rover traveling on the loose soil[J]. Journal of Mechanical Engineering, 2011, 47(23): 97-103.

[9] JU Hehua, CUI Pingyuan. Modelling and control for lunar rocker-bogie rover with slip[J]. Chinese Journal of Mechanical Engineering, 2005, 41(9): 134-139.

[10] GAO Haibo, ZHANG Peng, DENG Zongquan, et al. Development of suspension frame of new eight-wheel lunar rover[J]. Chinese Journal of Mechanical Engineering, 2008, 44(7): 85-92.

作者简介: 刘振, 男, 1983 年出生, 博士研究生。主要研究方向为星球 车低重力模拟。

E-mail: liuzhen_hit@163.com

高海波(通信作者), 男, 1970 年出生, 教授、博士研究生导师。主要研 究方向为移动机器人。

E-mail: gaohaibo@hit.edu.cn

邓宗全, 男, 1956 年出生, 教授, 博士研究生导师。主要研究方向为宇 航空间机构与控制。

E-mail: dengzongquan@hit.edu.cn

陶建国, 男, 1964 年出生, 教授, 博士研究生导师。主要研究方向为星 球探测车。

E-mail: taojianguo@hit.edu.cn 